directed against CGRP, were assessed in a phase 2 clinical trial NCT02192190 in patients with moderate to severe osteoarthritis $(\mathrm{OA})$ knee pain. Patients were randomized to placebo, galcanezumab $(5,25,120$ and $300 \mathrm{mg}$ subcutaneously every 4 weeks, at weeks 0 and 4 ) or celecoxib (200 mg once daily) for 16 weeks in a 2:1:1:1:1:1 ratio. The study was terminated after an interim analysis due to inadequate efficacy for OA pain.

Objectives: This study assessed the correlation of baseline plasma CGRP concentrations with signs, symptoms and radiographic severity of $\mathrm{OA}$, and response to galcanezumab and celecoxib treatments.

Methods: Plasma samples were collected at baseline and weeks 4, 8, 12 and 16 after study drug treatment. CGRP concentrations were determined by a validated high sensitivity (HS) assay. Correlation of baseline CGRP levels to WOMAC scores, PGA and radiographic Kellgren-Lawrence (K-L) grades were assessed using Spearman's correlation and Wilcoxon test. Patients were stratified into high vs low groups by baseline CGRP concentrations and post-treatment changes from baseline WOMAC scores evaluated by mixed effect model repeated measures for each subset.

Results: At the interim analysis, baseline plasma CGRP samples were available for 262 patients with 54 patients providing samples at study termination through the week 8 visit. The median CGRP concentration at baseline was $1.07 \mathrm{pg} / \mathrm{ml}$, range $<0.78$ to 33.91 , and $31 \%$ of patients were below the level of quantitation (BLQ, $<0.78 \mathrm{pg} / \mathrm{ml}$ ). Median baseline CGRP levels were $1.0 \mathrm{pg} / \mathrm{ml}$ for $\mathrm{K}-\mathrm{L}$ grade $2(\mathrm{~N}=178)$, and $1.2 \mathrm{pg} / \mathrm{ml}$ for $\mathrm{K}-\mathrm{L}$ grade $3(\mathrm{~N}=84)(\mathrm{p}=0.06)$. Correlations of WOMAC or PGA scores with baseline CGRP levels were all $r<0.01$ (showed no significant correlations). In OA patients receiving galcanezumab $300 \mathrm{mg} \mathrm{SC}$ at week 0 and week 4 , those with high baseline CGRP levels demonstrated a $14 \mathrm{~mm}$ improvement in WOMAC Pain response at week $12,(95 \% \mathrm{Cl} 0,29 \mathrm{~mm})$. The pain response to galcanezumab $300 \mathrm{mg}$ did not reach the magnitude of celecoxib response and no effects were seen at $5-120 \mathrm{mg}$ doses. Celecoxib treatment had larger pain reduction among patients with high baseline CGRP compared to low baseline CGRP levels. Treatment with celecoxib did not alter plasma CGRP concentrations.

Conclusions: At baseline, CGRP levels in OA patients were not associated with WOMAC or PGA scores. There was a modest association to radiographic K-L grade. Subgroup analyses of patients with high (>median) CGRP levels at baseline suggested a potential response to galcanezumab for the highest dose, $300 \mathrm{mg}$, but not lower doses. Celecoxib response was greater in those with higher CGRP levels. However, interpretation was limited by small samples sizes at the latter time points. Further studies may determine if enriching the OA population for higher CGRP levels at baseline, or if increased or longer dosing of galcanezumab would improve pain responses or if CGRP blockade is relevant in relieving OA knee pain. Disclosure of Interest: T. Mcnearney Shareholder of: Eli Lilly stock, Employee of: Eli Lilly and Company, C. Smith Shareholder of: Eli Lilly stock, Employee of: Eli Lilly and Company, R. Brown Shareholder of: Eli Lilly and Company, Employee of: Eli Lilly and Company, A. Camporeale Shareholder of: Eli Lilly stock, Employee of: Eli Lilly and Company, M. Deeg Shareholder of: Eli Lilly stock, Employee of: Eli Lilly and Company, D. Montieth Shareholder of: Eli Lilly stock, E. Collins Employee of: Eli Lilly and Company, T. Schnitzer Consultant for: Eli Lilly and Company, A. Kivitz: None declared, J. Talbot Shareholder of: Eli Lilly stock, Employee of: Eli Lilly and Company, Y. Jin Shareholder of: Eli Lilly stock, Employee of: Eli Lilly and Company

DOI: 10.1136/annrheumdis-2017-eular.2155

\section{SAT0549 ANTI-INFLAMMATORY PROPERTIES OF SM04690, A SMALL MOLECULE INHIBITOR OF THE WNT PATHWAY AS A POTENTIAL TREATMENT FOR KNEE OSTEOARTHRITIS}

V. Deshmukh, C. Barroga, M. Ibanez, T. Seo, S. Kc, Y. Yazici. Samumed, LLC, San Diego, CA, United States

Background: Osteoarthritis $(\mathrm{OA})$ is characterized by pain, deformity, and reduced function in the knee joint. Upregulated Wnt signaling affects the pathogenesis of $\mathrm{OA}$ through increased inflammation, increased subchondral bone and thinning cartilage. SM04690, a novel small molecule, was previously shown to inhibit the Wnt pathway and induce chondrogenesis in vitro and in vivo. ${ }^{1}$

Objectives: SM04690 was evaluated in preclinical studies to determine its capacity to reduce inflammation, and reduce pain in $\mathrm{OA}$.

Methods: Anti-inflammatory activity was evaluated by measuring cytokine (IL-6 and TNF- $\alpha$ ) secretion using ELISA with IL1-b stimulated synovial fibroblasts. A panel of pro-inflammatory cytokines (TNF- $\alpha$, IL-1 $\alpha$, IL-1 $\beta$, IL-2, IL-6, IL-8, IL-17A, IL-17F, IFN- $\gamma$ and PGE2) was evaluated by ELISA; $T$ and $B$ cell proliferation by flow cytometry in peripheral blood mononuclear cells (PBMCs), and T and B cell co-cultures stimulated with super-antigen (SAg) or lipopolysaccharides (LPS), compared to vehicle or benchmark immunosuppressant or steroid (cyclosporin $A$ and prednisolone). SM04690 effects on LPS-induced expression and phosphorylation of JNK, NFkB, Erk, cJun, Akt, Stat3 in THP-1 cells were measured by qPCR and Western Blot. In vivo SM04690 activity was evaluated in a rat monosodium iodoacetate (MIA) injection-induced OA model, immediately followed by a single intra-articular SM04690 or vehicle injection. Joint inflammation was evaluated by measuring synovial thickness and infiltrating cells histology; inflammatory cytokines (IL-1 $\alpha$, IL-1 $\beta$, IL-2, IL-5, IL-6, IL-8, TNF- $\alpha$ and IFN- $\gamma$ ) by qPCR and ELISA and cartilage protection by qPCR for matrix metalloproteinases (MMPs). Pain was measured as paw withdrawal threshold using Von Frey apparatus.
Results: SM04690 inhibited IL-1b-induced TNF- $\alpha$ and IL-6 secretion in synovial fibroblasts $\left(E_{50} @ 30 \mathrm{nM}\right)$. SM04690 significantly inhibited $(p<0.01) \mathrm{SAg}$ and LPS stimulated pro-inflammatory cytokine production (TNF- $\alpha$, IL1- $\alpha$, IL1- $\beta$, IL-2, IL-6, IL-8, IL-17A, IL-17F, IFN- $\gamma$, PGE2), T and B cell proliferation in PBMCs and $T$ and $B$ cell co-cultures (Figure $A$ ), with activity comparable to or better than cyclosporin A and prednisolone. SM04690 treatment specifically decreased LPS-induced gene expression $(p<0.01)$ and phosphorylation of NFkB in THP-1 cells with no effect on JNK, Erk, cJun, Akt and Stat3. Compared to vehicle in the rat MIA OA model, SM04690 injection reduced inflammatory cells, decreased synovial thickness $(p<0.05$,Figure $B)$, inhibited production of pro-inflammatory cytokines and MMPs $(p<0.05)$. SM04690 increased $(p<0.01)$ paw withdrawal threshold in treated rats compared to vehicle at multiple time points (Figure $\mathrm{C}$ ).

Figure. SM04690 inhibited inflammatory cytokine production in vitro and decreased inflammation and pain in the MIA model of OA
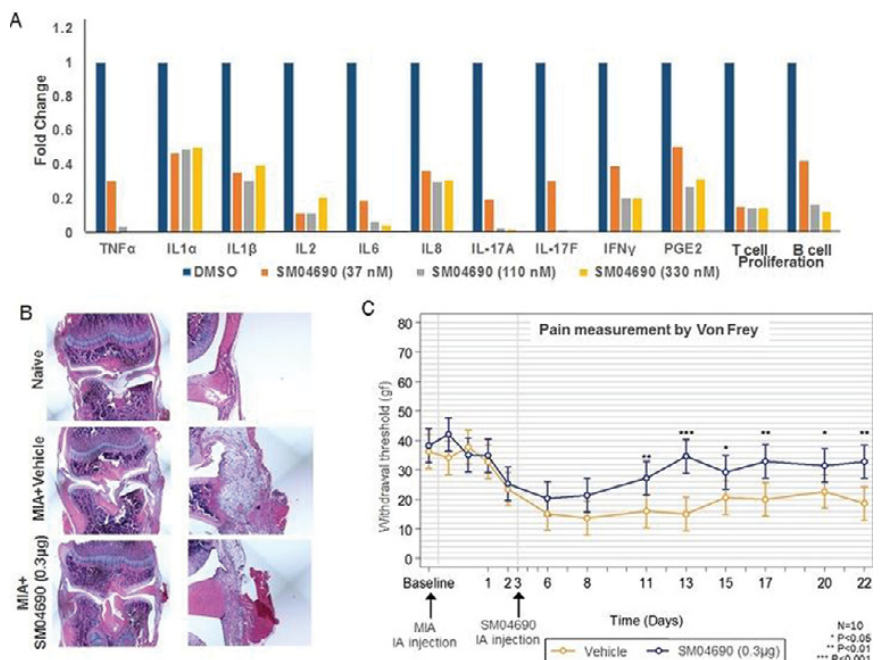

Conclusions: SM04690 demonstrated potent anti-inflammatory properties, comparable to or greater than cyclosporin $A$ and prednisolone. In a rat knee $O A$ model, SM04690 injection reduced inflammation, protease production and pain compared to vehicle. The anti-inflammatory properties of SM04690 may provide beneficial effects in the treatment of OA. Clinical studies are ongoing.

References:

[1] Hood et al. OAC 2016, s187.

Disclosure of Interest: V. Deshmukh Shareholder of: Samumed, LLC, Employee of: Samumed, LLC, C. Barroga Shareholder of: Samumed, LLC, Employee of: Samumed, LLC, M. Ibanez Shareholder of: Samumed, LLC, Employee of: Samumed, LLC, T. Seo Shareholder of: Samumed, LLC, Employee of: Samumed, LLC, S. KC Shareholder of: Samumed, LLC, Employee of: Samumed, LLC, Y. Yazici Shareholder of: Samumed, LLC, Employee of: Samumed, LLC DOI: 10.1136/annrheumdis-2017-eular.6460

\section{SAT0550 NEUROPATHIC PAIN AMONG PATIENTS WITH PRIMARY KNEE OSTEOARTHRITIS- RESULTS OF A CROSS SECTIONAL STUDY FROM A TERTIARY CARE CENTRE IN SOUTHERN INDIA}

V. Narayan R ${ }^{1}$, M.M. Thabah ${ }^{2}$, M. Poduval ${ }^{3} .{ }^{1}$ MBBS Student; ${ }^{2}$ Medicine; ${ }^{3}$ Orthopedic Surgery, Jawaharlal Institute of PostgraduateMedical Education and Research, Puducherry, India

Background: Pain in osteoarthritis $(O A)$ is generally believed to be nociceptive because of local structural pathology and joint failure which so characterizes OA. However, there is often a discordance between the radiographic knee OA and pain, suggesting that the pain can be contributed by other mechanisms other than nociception. ${ }^{1}$ Recently it is shown that pain in knee OA may have a neuropathic component. $^{2}$

Objectives: The objective of the study was to assess the level of neuropathic pain in patients with knee $\mathrm{OA}$, and identify the clinical and socio-demographical factors associated with neuropathic pain

Study design: Cross sectional study.

Setting: Medicine and Orthopaedic outpatient department (OPD) of a tertiary care centre located in southern India

Methods: One-hundred and sixty-one patients with knee OA satisfying the American College of Rheumatology 1986 clinical and radiographic classification criteria for knee OA were studied. Neuropathic pain was assessed by the Douleur Neuropathique in 4 questions (DN4) questionnaire; ${ }^{3}$ score of $4 / 10$ or more was classified as diagnostic for neuropathic pain. The Indian version of Western Ontario and McMaster Universities Osteoarthritis Index (WOMAC) ${ }^{4}$ was used to assess pain, stiffness and physical function of the patients. Factors associated with neuropathic pain were explored.

Results: Mean age was $55.7 \pm 8.8$ years. The mean total Indian WOMAC was 